\title{
List Mode-Driven Cardiac and Respiratory Gating in PET
}

Florian Büther ${ }^{1}$, Mohammad Dawood ${ }^{2,3}$, Lars Stegger ${ }^{1}$, Frank Wübbeling ${ }^{4}$, Michael Schäfers ${ }^{1,3}$, Otmar Schober ${ }^{1,3}$, and Klaus P. Schäfers ${ }^{1,3}$

${ }^{1}$ Department of Nuclear Medicine, University of Münster, Münster, Germany; ${ }^{2}$ Department of Computer Science, University of Münster, Münster, Germany; ${ }^{3}$ European Institute of Molecular Imaging, University of Münster, Münster, Germany; and ${ }^{4}$ Department of Computational and Applied Mathematics, University of Münster, Münster, Germany

Gating methods acquiring biosignals (such as electrocardiography [ECG] and respiration) during PET enable one to reduce motion effects that potentially lead to image blurring and artifacts. This study evaluated different cardiac and respiratory gating methods: one based on ECG signals for cardiac gating and video signals for respiratory gating; 2 others based on measured inherent list mode events. Methods: Twenty-nine patients with coronary artery disease underwent a 20-min ECG-gated single-bed list mode PET scan of the heart. Of these, 17 were monitored by a video camera registering a marker on the patient's abdomen, thus capturing the respiratory motion for PET gating (video method). Additionally, respiratory and cardiac gating information was deduced without auxiliary measurements by dividing the list mode stream in 50-ms frames and then either determining the number of coincidences (sensitivity method) or computing the axial center of mass and SD of the measured counting rates in the same frames (center-of-mass method). The gated datasets (respiratory and cardiac gating) were reconstructed without attenuation correction. Measured wall thicknesses, maximum displacement of the left ventricular wall, and ejection fraction served as measures of the exactness of gating. Results: All methods successfully captured respiratory motion and significantly decreased motion-induced blurring in the gated images. The center-of-mass method resulted in significantly larger left ventricular wall displacements than did the sensitivity method $(P<$ 0.02 ); other differences were nonsignificant. List mode-based cardiac gating was found to work well for patients with high ${ }^{18} \mathrm{~F}-\mathrm{FDG}$ uptake when the center-of-mass method was used, leading to an ejection fraction correlation coefficient of $r=0.95$ as compared with ECG-based gating. However, the sensitivity method did not always result in valid cardiac gating information, even in patients with high ${ }^{18} \mathrm{~F}-\mathrm{FDG}$ uptake. Conclusion: Our study demonstrated that valid gating signals during PET scans cannot be obtained only by tracking the external motion or applying an ECG but also by simply analyzing the PET list mode stream on a frame-by-frame basis.

Key Words: list mode PET; respiratory motion; respiratory gating; cardiac gating

J Nucl Med 2009; 50:674-681

DOI: 10.2967/jnumed.108.059204

Received Oct. 17, 2008; revision accepted Jan. 14, 2009.

For correspondence or reprints contact: Florian Büther, Department of Nuclear Medicine, University of Münster, Albert-Schweitzer-Strasse 33, 48149 Münster, Germany.

E-mail: butherf@uni-muenster.de

COPYRIGHT ๑ 2009 by the Society of Nuclear Medicine, Inc.
$\mathbf{P}$ in the clinical the clinical fields of oncology, neurology, cardiology, and several others. PET can show functional, metabolic, and molecular processes in vivo with a high sensitivity and offers the unique feature of absolute quantification of radiotracer distribution. However, several mathematic corrections have to be applied to the measured PET raw data before or during image reconstruction to obtain absolute quantitative data. The most important of these is attenuation correction, that is, correcting for the loss of coincidence photons due to absorption while they are traversing the human body. Accurate attenuation correction requires knowledge of attenuation values in the field of view of the scanner. In stand-alone PET scanners, this information is acquired during an additional transmission scan using external $\gamma$-sources to obtain attenuation values for every measured line of response.

An important milestone in PET history was the development of scanners combining PET and x-ray-based CT (1). Apart from acquiring both functional/molecular and morphologic information in a single scanning session, leading to a higher diagnostic efficiency and improved spatial coregistration, PET attenuation correction can, in principle, be improved by replacing the slow and lowcounting-rate transmission scan using external radioactive sources by a fast and high-counting-rate CT scan. The result, higher patient throughput and anatomic landmark information, significantly improves the signal-to-noise ratio of the attenuation correction map (2). However, PET wholebody emission scans typically require several minutes per bed position whereas the CT scan is usually acquired within a few seconds. During PET acquisitions, patients cannot hold their breath long enough (as is possible during the CT scan), resulting in motion-blurred PET data due to respiratory movement, especially in thoracic and abdominal areas. Furthermore, if the CT data are not acquired in a respiration phase that fits the mean PET respiration phase, spatial coregistration between CT and PET is impaired. Thereby, an erroneous assignment of tissue attenuation may occur, leading to attenuation correction artifacts (3). Additionally, 
the heart itself moves because of cardiac contractions, thereby leading to an additional increase in motion blur.

One method to resolve this problem is based on gating the PET data: the whole set of PET raw data is distributed in subsets representing the different phases of cardiac or respiratory motion. Reconstruction of the subsets leads to image datasets with greatly reduced motion, albeit with lower statistics $(3,4)$. Although gating with respect to cardiac contraction is usually achieved by using an electrocardiography (ECG) monitor (which is already incorporated in some scanner designs), different technical strategies have been developed to implement respiratory gating, for example, use of a sensor on the patient's abdomen to detect pressure differences due to respiration (4), infrared (5) or optical (6) video camera systems to track thoracic motion during the PET scans, piezoelectric crystals (7), or sensors that detect the temperature of air flowing into the lungs (8). However, all these methods rely on additional equipment that measures only external motion, which may not necessarily correlate well with internal organ motion (9). Therefore, it would be desirable to implement gating methods based directly on the measured PET emission data. We have developed and implemented 2 purely event-based methods for human cardiac PET/CT and compared their performance with video-based and ECG-based gating methods.

\section{MATERIALS AND METHODS}

\section{PET/CT Scanner}

PET data in this study were acquired on a list mode-capable PET/CT scanner (Biograph Sensation 16; Siemens AG Medical Solutions). The PET scanner comprises 24 detector rings of lutetium oxyorthosilicate detector crystals (384 crystal elements per ring; ring diameter of $824 \mathrm{~mm}$ ). The axial field of view is 162 $\mathrm{mm}$; the transaxial field of view, $585 \mathrm{~mm}$. The scanner is operated in 3-dimensional mode using axial compression (5 segments with a span of 7), resulting in sinogram datasets of $192 \times 192 \times 175$ bins. If operated in list mode, the measured coincidences (prompt and delayed) and time tags are saved in a file that can be processed retrospectively. The scanner is equipped with an internal ECG device that allows recording of the R-wave temporal position to a trigger file while operating in list mode.

\section{Patient Data and Preparation}

This study included data from 29 patients who were referred for an ${ }^{18}$ F-FDG PET scan for routine evaluation of myocardial viability. Patients with a heart rate higher than 65 beats/min received $\beta$-blocker medication before the scans to slow the heart rate. Before and during the ${ }^{18} \mathrm{~F}$-FDG uptake phase, patients underwent a hyperinsulinemic euglycemic clamp technique to increase ${ }^{18} \mathrm{~F}-\mathrm{FDG}$ uptake in the heart (10). An intravenous dose of $4 \mathrm{MBq}$ of ${ }^{18} \mathrm{~F}$-FDG per kilogram of body weight was injected $1 \mathrm{~h}$ before the PET scan.

\section{Data Acquisition}

During the PET/CT scan, the patients were positioned supine with the arms elevated behind the head. The heart was positioned inside the scanner field of view with the help of a CT topogram. After standard clinical PET and CT scans had been acquired, a 20-min list mode PET scan (single bed position) was initiated while the heart beat was monitored using the scanner's ECG device. To gate the PET list mode data with respect to respiratory motion, a video camera installed at the end of the patient bed monitored the movement of a white circular spot located on a black disk that was mounted on a flexible belt around the patient's abdomen in 17 of the 29 scans (video method, Fig. 1) (6). Synchronization with the list mode scan was ensured by a light-emitting diode attached to the black disk, emitting light only during the list mode scan. The video signal was transferred to a personal computer notebook, where purpose-built in-house software analyzed the incoming signal in real time. Briefly, the video image of the black disk was segmented using a simple threshold method to discriminate between black background and white spot. Then, the vertical coordinate of the center of the mass of the white spot was calculated and stored, resulting in a breathing curve (sample time, $200 \mathrm{~ms}$ ) that can be used for retrospective gating (Supplemental Fig. 1A; supplemental materials are available online only at http:// jnm.snmjournals.org).

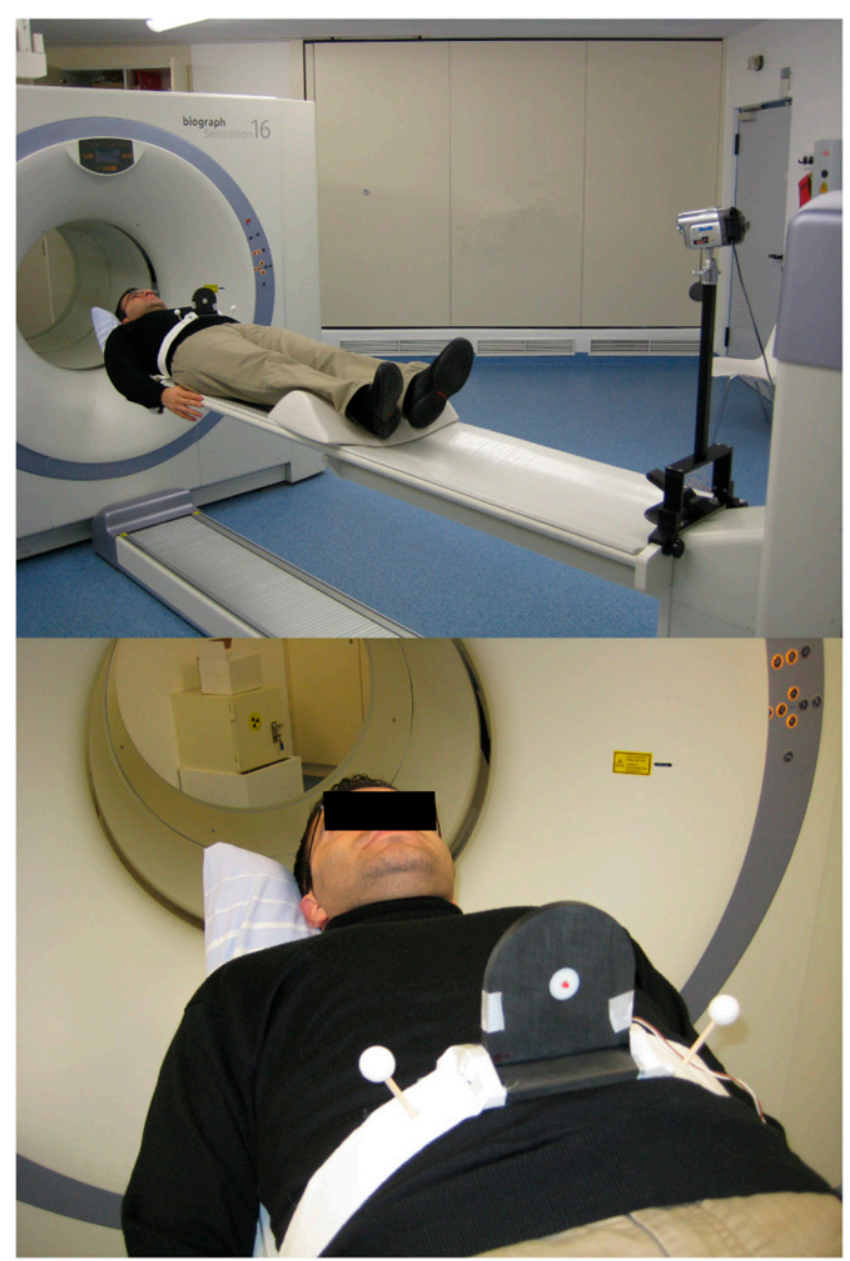

FIGURE 1. Setup of video camera system. Video camera at end of bed tracks motion of white spot on black disk mounted on belt around patient. Light-emitting diode in center of spot starts lighting when scan begins, thus synchronizing with PET list mode stream. 


\section{Data-Driven Gating and Data Reconstruction}

Two gating methods (sensitivity and center of mass) that are purely list mode-based were applied to all 29 patient scans to derive information on respiratory and cardiac motion (Fig. 2). The sensitivity method, derived from He et al. (11), makes use of the fact that the geometric sensitivity profile (i.e., the probability that an annihilation event located at a certain point inside the scanner is potentially detected) of the PET scanner is inhomogeneous (Fig. 2A), as oblique coincidences can be measured only in the center of the scanner's axial field of view, not at the front or rear of the scanner's axis. Therefore, a moving activity source inside the scanner's field of view will result in slightly different numbers of measured coincidences in small time frames. To apply this method, we retrospectively analyzed the resulting list mode file by counting the numbers of prompt and delayed coincidences in 50-ms frames $(P(t)$ and $D(t)$, respectively). Because the physical decay of the radionuclide leads to a decrease in counting rate for later frames, the determined counting rates were corrected for this decay by weighting every measured coincidence with the exponential time-dependent factor $\exp (\lambda t)\left(\lambda\right.$ : decay constant of $\left.{ }^{18} \mathrm{~F}\right)$. The difference in decay-corrected prompt and delayed counting rates is an estimate of the true coincidence rate $T(t)$; thus, this difference was calculated:

$$
T(t)=(P(t)-D(t)) \cdot \exp (\lambda t)
$$

This calculation results in a raw respiration curve that, because of the statistical nature of the radioactive decay, is comparatively noisy (Fig. 3A). To extract motion information, we transformed the curve into the frequency domain using the fast Fourier transformation FFT (Fig. 3B):

$$
\widetilde{T}(f)=F F T[T(t)]
$$

Typically, 2 prominent components can then be identified in the frequency spectrum $|\tilde{T}(f)|$ : a low-frequency contribution $\tilde{T}_{\text {resp }}(f)$ caused by respiratory motion, usually limited to absolute values smaller than $0.5 \mathrm{~Hz}$, and a contribution $\tilde{T}_{\text {card }}(f)$ caused by heart contractions centered around frequencies $f_{\text {card }}$ and $-f_{\text {card }}$. These 2 components were isolated by setting all other frequencies to 0 (Figs. 3C and 3E) and then transformed back into the time domain using the inverse fast Fourier transformation iFFT, resulting in
2 gating curves $T_{\text {resp }}(t)=i F F T\left[\tilde{T}_{\text {resp }}(f)\right]$ and $T_{\text {card }}(t)=$ $i F F T\left[\tilde{T}_{\text {card }}(f)\right]$. This leads to smooth curves valid for subsequent gating (Figs. 3D and 3F and Supplemental Fig. 1B).

The center-of-mass method is based on a more direct estimation of the motion inside the field of view (Fig. 2B). For this method, the true coincidence counting rate per $50-\mathrm{ms}$ frame was determined from the list mode file as a function of the axial coordinate (slice number). Oblique coincidence events, that is, coincidences between detectors located on different rings, were rebinned into the 47 transverse sinograms of the Biograph Sensation 16 scanner with the help of the single-slice rebinning algorithm (12), thus enabling an axial assignment of coincidence events along the scanner's $z$-axis. The resulting function is essentially an axial histogram $T(i, t)$ ( $t$ : time frame; $i$ : slice number; $i=1, \ldots, 47)$ of measured true events within time frame $t$. As activity inside the thorax moves along this axis because of respiratory motion or cardiac contractions, the histogram that belongs to a certain time frame contains information about the respiratory and cardiac phase. To extract this information, the axial center of mass as a function of time frame center of mass $(t)$ of every histogram $T(i, t)$ was computed as:

$$
\text { Center of mass }(t)=\frac{\sum_{i} i \cdot T(i, t)}{\sum_{i} T(i, t)} .
$$

Additionally, for cardiac gating purposes, the $\mathrm{SD} S D(t)$ has also been computed as:

$$
S D(t)=\sqrt{\frac{\sum_{i} i^{2} \cdot T(i, t)}{\sum_{i} T(i, t)}-\text { center of } \operatorname{mass}(t)^{2} .}
$$

This SD method is valuable in some cases because the heart contraction does not always correlate with a translational shift along the $z$-axis but sometimes correlates with a change in axial variance of the histograms. These computations resulted in raw respiration curves that were filtered in the same way as described above. Similar to the sensitivity method, this procedure results in smooth curves center of mass resp $(t)$ (Supplemental Fig. 1C), center of mass card $(t)$, and $S D_{\text {card }}(t)$ (not shown) suited for gating purposes.
A

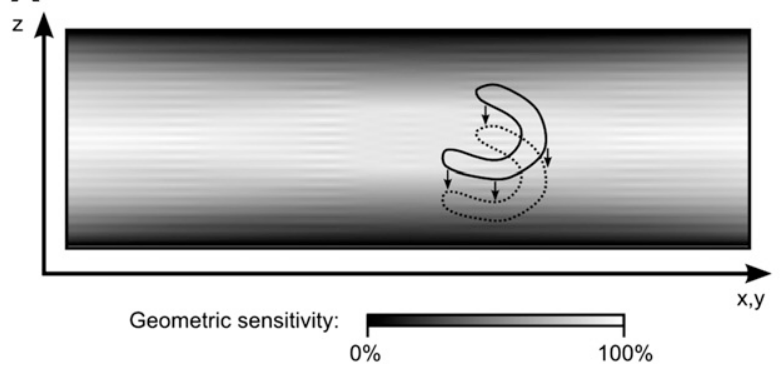

B

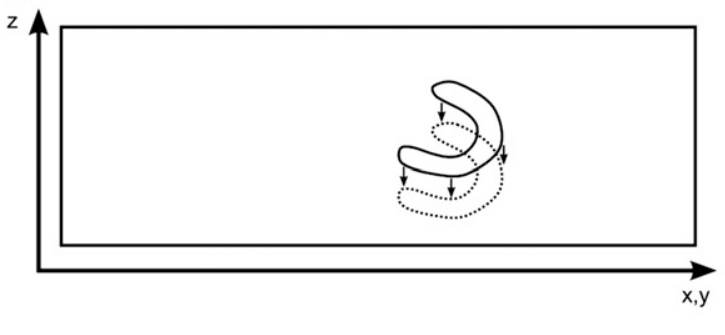

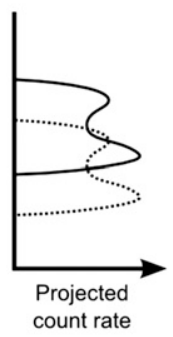

FIGURE 2. Purely event-driven gating methods. (A) Moving activities inside field of view will experience different detection probabilities due to inhomogeneous geometric sensitivities; thus, coincidence counting rates vary according to respiratory motion (sensitivity method). (B) Activities moving along z-axis shift axial profiles and thus center of mass of coincidence counting rates, indicating respiratory motion (center-of-mass method). 

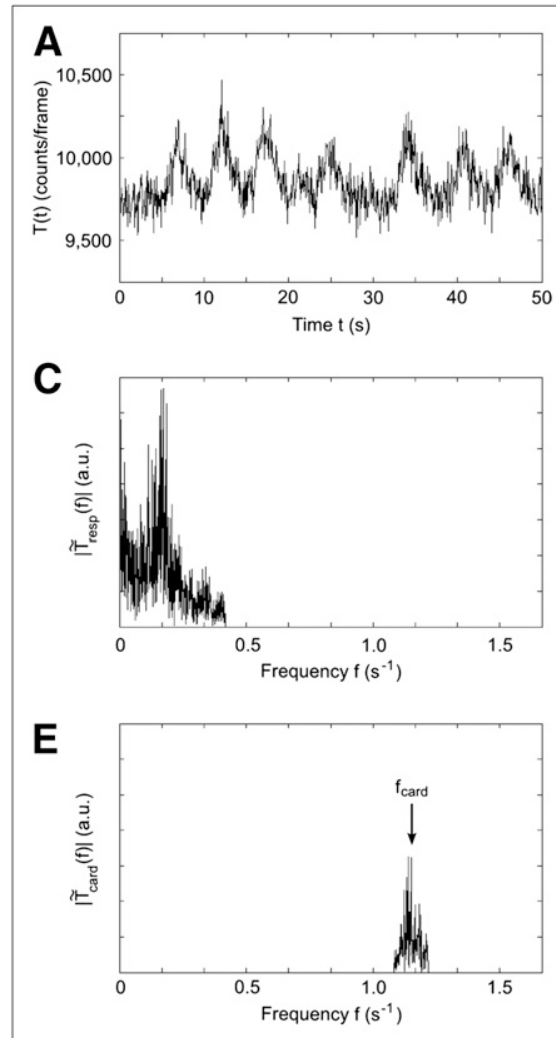

B
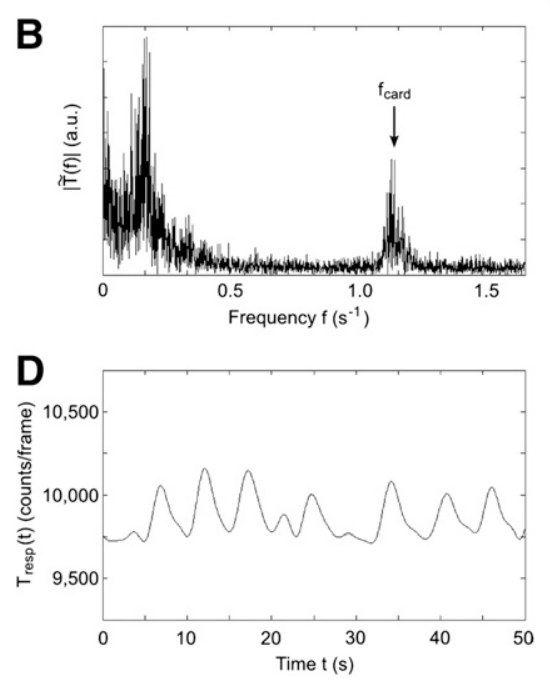

$\mathbf{F}$

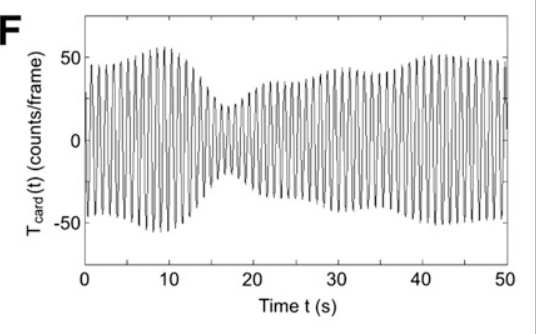

FIGURE 3. Concept of Fourier filtering of raw respiratory curves: typical raw respiratory curve (A); Fourier spectrum of raw curve (only positive frequencies are shown here) (B); isolated respiratory and cardiac components of spectrum (C and $\mathrm{E}$, respectively); filtered respiratory and cardiac curves derived by inverse Fourier transformation ( $\mathrm{D}$ and $\mathrm{F}$, respectively).
Similarity between the obtained respiratory curves was measured computing Spearman rank correlation coefficient $\rho$.

An amplitude-based approach was applied rather than a timebased approach to derive respiratory gating intervals from the breathing curves, as amplitude-based techniques were shown to capture respiratory motion more accurately than time-based techniques $(6,13)$. To obtain gates with comparable noise, the gating intervals were determined to result in 8 gates with equal measured coincidences (variable amplitude-based gating). This variable division of amplitudes avoids gates with limited amounts of measured events, making subsequent image analysis easier (6). Furthermore, this division is better suited for comparison of different gating methods, as one cannot necessarily assume a linear relation between acquired respiratory curves for different methods; instead, monotonic relations are to be expected at best, as different physical quantities are measured.

Because no absolute heart phase measurement is available in the case of ECG-based gating, a time-based gating scheme was chosen for the cardiac gating methods. Additionally, whereas the list mode-based gating methods potentially measure absolute information on heart phase, the amplitudes derived from these methods are characterized by beat waves because of frequency band limitations in the filtering step described above (Fig. 3F). For the time-based gating, the maxima of the obtained cardiac curves were determined; the time interval between 2 maxima was then divided into 10 equidistant cardiac gates. This division was done with the obtained ECG data as well; however, only R-R intervals representing pulse frequencies between 50 and 80 beats/min were accepted, to minimize potential R-wave misinterpretations or extra systoles. These misinterpretations and extra systoles can easily be identified by interval lengths that are significantly separated from the mean peak in R-R interval length histograms.
The PET list mode file (typically $\sim 1.5$ GB of PET coincidence raw data) was binned into gated sinogram datasets according to the determined gating schemes using purpose-built in-house software. Applied corrections included decay and dead-time corrections as well as detector normalization. For comparison, a nongated sinogram dataset using the whole set of PET list mode data was additionally generated.

The open-source package Software for Tomographic Image Reconstruction (available at http://stir.sourceforge.net/) was used to reconstruct the preprocessed sinogram sets without attenuation correction. An iterative 3-dimensional ordered-subsets expectation maximization algorithm was applied to obtain the PET images. The reconstruction was performed using 4 subsets and 5 iterations. A postreconstruction gaussian filter $(4 \mathrm{~mm}$ in full width at half maximum) was finally applied to the images. The obtained images comprised $175 \times 175 \times 47$ cubic voxels with an edge length of $3.375 \mathrm{~mm}$.

\section{Data Analysis}

The displacement of the left ventricular wall between gate 1 and gate 8 (representing maximum inspiration and maximum expiration) was chosen as a measure of successfully capturing the respiratory motion. This choice was made because large respiratory shifts found for a given gating method indicate better performance in capturing the respiratory motion than do methods yielding smaller shifts. This motion shift was determined using the automatic reslicer of the MPI-Tool program (Advanced Tomo Vision $\mathrm{GmbH}$ ). The segmented left ventricular wall in the maximum-inspiration image was iteratively shifted against the maximum-expiration image until a maximum in correlation coefficient was reached. The result was a motion resolution of approximately $1 \mathrm{~mm}$. 
Another approach taken toward evaluating the success of respiratory gating was measurement of cardiac wall thicknesses, because respiratory motion contributes to partial-volume effects due to thin myocardial walls, leading to motion-dependent blurring of ventricular walls and thus overestimated measured thicknesses. For this purpose, the thickness of the anterior left ventricular wall in the nongated and gated PET images was determined, as this is the part of the heart that is best seen in nonattenuation-corrected PET images; additionally, this region in general is significantly affected by respiratory motion effects, since the direction of respiratory motion is usually almost perpendicular to the anterior wall, as opposed to the lateral and septal walls. For measuring left ventricular wall thickness, the heart was realigned into short-axis images. The thickness of the anterior wall was then determined as the mean of the full width at half maximum of gaussian profile fits taken at 3 subsequent midventricular slices. For the gated images, this measurement was done for the end-expiration gate, leading to 4 thickness values in total for each patient.

The quality of cardiac gating was determined by measuring the left ventricular ejection fraction derived from the different gating methods, using validated purpose-built in-house software $(14,15)$. In general, with the different gating methods, larger values of ejection fraction reflect a comparatively better-quality resolving of cardiac contractions. It is clear that in cases where the ${ }^{18} \mathrm{~F}-\mathrm{FDG}$ uptake in the left ventricle is low, limited information about cardiac contractions from the list mode stream can be expected; therefore, only scans with sufficiently high ${ }^{18} \mathrm{~F}-\mathrm{FDG}$ uptake in the left ventricle were included in the ejection fraction analysis.

Two-tailed Wilcoxon signed rank tests were applied to investigate significant differences between the 3 methods; $P$ values of less then 0.05 were considered to represent significant differences.

\section{RESULTS}

A comparison of the obtained respiration curves revealed a striking similarity between all 3 methods (Supplemental Fig. 1). Especially, the position of respiratory maxima (maximum inspiration) in time coincided for most acquired curves. The video method gave the smoothest curves, whereas the other methods typically resulted in noisier curves due to the inherent statistical nature of radioactive decay. The correlation analysis revealed considerable mean correlations of $0.65,0.68$, and $0.71(n=17$; Spearman rank correlation coefficient $\rho$ for correlation between the video and sensitivity methods, the video and center-of-mass methods, and the sensitivity and center-of-mass methods, respectively).

Visual comparison of the gated, non-attenuation-corrected PET images showed almost no difference between the 3 respiratory gating methods in terms of visible motion shifts. A typical outcome using list mode-based gating is shown in Figure 4.

The analysis of maximum displacement due to respiration revealed similar mean displacements of the left ventricular wall for the 3 methods: $8.1,7.6$, and $8.8 \mathrm{~mm}$ for the video, sensitivity, and center-of-mass methods, respectively ( $n=17$; Table 1). A statistical analysis demonstrated no significant difference between the video and center-of-mass
A
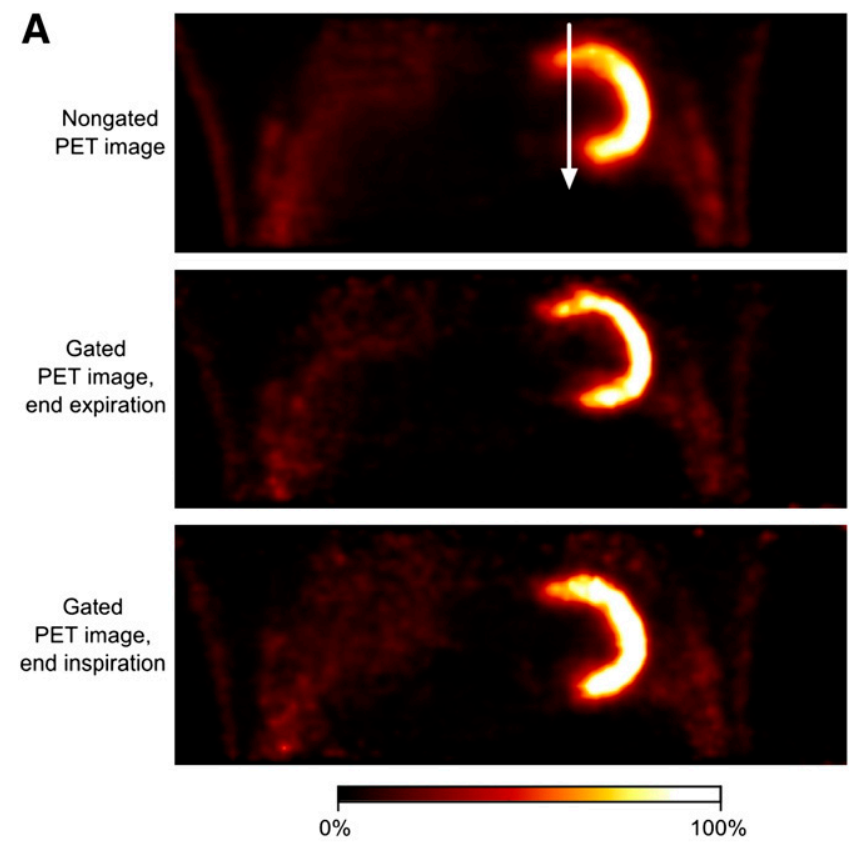

B

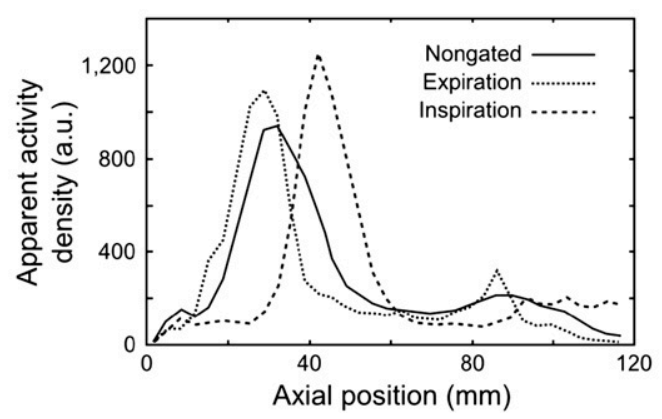

FIGURE 4. (A) Coronal view of computed nongated and gated PET images using center-of-mass method. Video and sensitivity methods gave visually almost identical images in this patient. (B) Profiles along arrow in A reveal respiratory motion of heart and demonstrate superior resolution of gated images. a.u. = arbitrary units.

methods or between the video and sensitivity methods; however, use of the center-of-mass method resulted in a significantly larger displacement than did use of the sensitivity method $(P<0.02)$. The linear correlation coefficient reached a maximum value of $r=0.93$ between the video and center-of-mass methods and was lowest (0.85) between

TABLE 1. Mean Respiratory Displacement of Left Ventricular Wall Between Gate 1 and Gate 8 (Maximum Expiration and Maximum Inspiration, Respectively)

Mean displacement \pm SD $(\mathrm{mm})$

Respiratory gating method

Video

$n=17$

$8.1 \pm 5.0$

$7.6 \pm 4.6$

Center of mass $\quad 8.8 \pm 4.3$ $n=29$ $8.4 \pm 4.6$ $9.4 \pm 4.5$ 
the video and sensitivity methods; comparing the sensitivity and center-of-mass methods revealed an $r$ of 0.91 . Mean values for the sensitivity and center-of-mass methods in the whole patient collective $(n=29)$ were 8.4 and $9.4 \mathrm{~mm}$, respectively, with a highly significant difference $(P<0.01)$ and a linear correlation of $r=0.90$.

A decrease in motion-induced blurring when respiratorygated PET data were used was demonstrated by wall thickness analysis (Table 2; a typical outcome is shown in Supplemental Fig. 2). Despite the applied clamp technique, left ventricular ${ }^{18} \mathrm{~F}$-FDG uptake was too low in 2 patients to reasonably determine wall thicknesses; therefore, the number of evaluated scans was reduced to 15 . The mean thicknesses of the left ventricular wall were $18.0 \mathrm{~mm}$ in nongated PET images, $16.2 \mathrm{~mm}$ in video-based images, $15.8 \mathrm{~mm}$ in sensitivity-based images, and $15.8 \mathrm{~mm}$ in center-of-mass-based images. All 3 gating methods resulted in a highly significant decrease in thickness, compared with the nongated data $(P<0.002)$. There were no significant differences in wall thickness between the gating methods. In the whole patient collective $(n=26 ; 3$ scans showed too low an ${ }^{18} \mathrm{~F}-\mathrm{FDG}$ uptake in the left ventricular myocardium), the mean values for nongated thicknesses and thicknesses based on sensitivity and center of mass were 17.9, 15.6, and $15.5 \mathrm{~mm}$, respectively; the gated values were significantly smaller than the nongated values $(P<0.0001)$; again, no statistical difference was found between the sensitivity and center-of-mass methods.

Using only scans with sufficiently high tracer uptake in the heart excluded 8 scans of 29 for the cardiac gating analysis; none of the list mode-based gating methods showed a cardiac peak in the Fourier spectrum in these cases. Correlation plots of the measured ejection fractions using list mode-based gating versus ECG-based ejection fraction, as well as a typical outcome for a list modebased cardiac gated PET image, are shown in Figure 5. There were cases in which a given list mode-based gating method did not lead to valid gating, even though the corresponding ${ }^{18} \mathrm{~F}-\mathrm{FDG}$ uptake was comparatively high, resulting in rather small linear correlation coefficients compared with ECG-based gating $(0.41,0.61$, and 0.59 for sensitivity-based gating, center-of-mass-based gating, and SD-based gating, respectively). However, combining the latter 2 methods (as they are essentially the same method) by taking the larger ejection fraction value resulted in a

\begin{tabular}{|c|c|c|}
\hline \multirow[b]{2}{*}{ Respiratory gating method } & \multicolumn{2}{|c|}{$\begin{array}{l}\text { Mean wall thickness } \pm S D \\
\qquad(\mathrm{~mm})\end{array}$} \\
\hline & $n=15$ & $n=26$ \\
\hline No gating & $18.0 \pm 3.6$ & $17.9 \pm 3.2$ \\
\hline Video & $16.2 \pm 3.3$ & - \\
\hline Sensitivity & $15.8 \pm 2.0$ & $15.6 \pm 1.9$ \\
\hline Center of mass & $15.8 \pm 3.0$ & $15.5 \pm 2.5$ \\
\hline
\end{tabular}

better correlation $(r=0.89)$ and a linear regression function $\mathrm{f}(x)=0.90 x+0.03 \%$, which is close to identity (Fig. 5B). Still, the difference in ejection fraction between ECG-based and center-of-mass-based gating is highly significant, at $P$ less than 0.002 (mean \pm SD: $49.6 \% \pm$ $13.4 \%$ and $44.6 \% \pm 13.5 \%$ for ECG-based and center-ofmass-based gating, respectively). The quality of list mode-based cardiac gating clearly depended on the apparent ${ }^{18}$ F-FDG uptake in the heart; taking only those scans with high tracer uptake into account (further reducing the number of evaluable scans to 15) generally leads to improved correlation coefficients, which in the case of the center-of-mass approach was determined as $r=0.95$ with a linear regression function $\mathrm{f}(x)=0.95 x-0.21 \%$ (Fig. 5B). However, ejection fractions in this case $(48.8 \% \pm$ $15.8 \%$ for ECG-based and $45.5 \% \pm 15.6 \%$ for center-ofmass-based gating) were still significantly different, at a $P$ value of 0.025 .

\section{DISCUSSION}

Respiratory motion-related image artifacts are known to be a source of erroneous tracer quantification in cardiac PET/CT. It is therefore desirable to eliminate these motioninduced effects from the images. Methods besides gating techniques have been proposed for this task, including the acquisition of a slow CT scan over several respiratory cycles that better fits the respiratory-blurred PET data (16) or the use of an averaged CT scan derived from 4-dimensional CT measurements (17), therefore simulating a stand-alone PET scan, avoiding major attenuation correction artifacts, and retaining the whole PET statistics. These methods, however, still lead to motion-blurred data, resulting in a potential effective loss of resolution and of quantitative information from the images. Furthermore, it is not clear whether a limited number of acquired CT respiratory phases really corresponds well to the PET data.

Respiratory gating avoids blurring by dividing the PET coincidence dataset into smaller data subsets representing just a single respiratory phase. Crucial to this technique is the recording of respiratory motion as a function of scanning time. In this feasibility study, we have shown that 2 gating methods that are driven purely by list mode events and do not rely on auxiliary measurements of respiratory motion are adequate for cardiac PET/CT studies. They compare most favorably with traditional video-based gating.

Quantitatively, video-based gating and center-of-massbased gating perform similarly in measured motion shifts of the left ventricle, whereas sensitivity-based gating performs slightly but significantly worse than the other 2 methods. This poorer performance may be due to the fact that the center-of-mass method is based on, essentially, measuring the internal craniocaudal motion of the heart itself, as opposed to the sensitivity method, which is not at all based on a direct measurement of motion. The insignificant difference between the video and center-of-mass methods is 

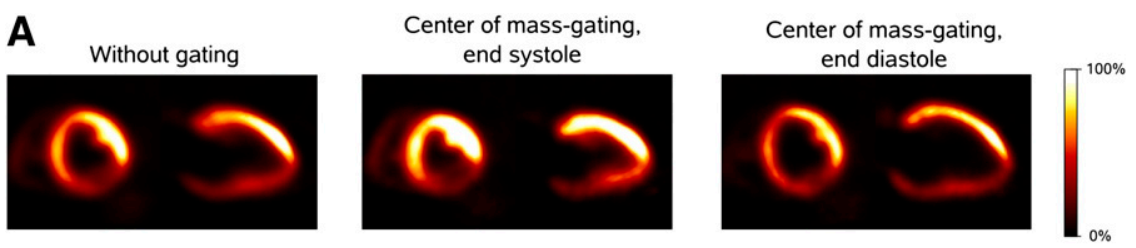

B
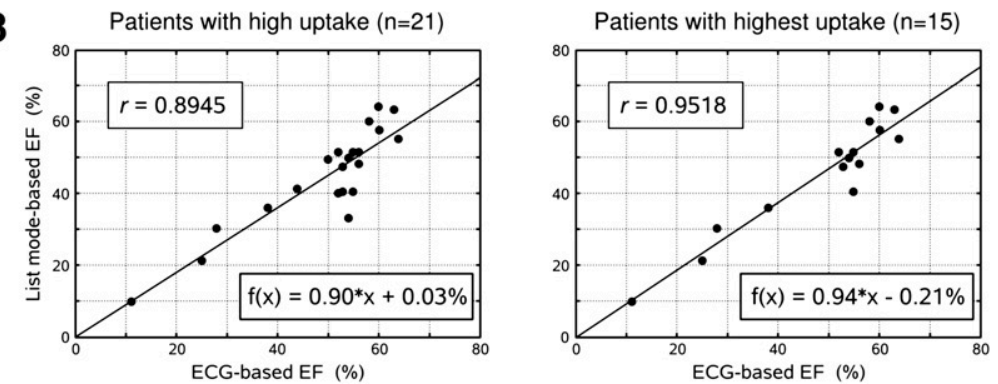

FIGURE 5. List mode-based cardiac gating. (A) In typical example using center-of-mass method, cardiac contraction is clearly resolved. (B) Correlation plots of ejection fraction using list mode-based gating methods (center of mass) vs. ECG-based gating for patients with highest tracer uptake. EF = ejection fraction.

consistent with results from MRI studies correlating internal motion of organs with external skin motion, revealing a clear correlation between the two (18).

It is both surprising and helpful that information not only about respiratory motion but also about heart contraction can additionally be extracted directly from the PET emission data. It is, however, clear that this extraction essentially requires sufficient tracer uptake in the heart. The results presented here indicate that up to now, list modebased cardiac gating usually results in smaller ejection fractions than ECG-based gating; this result is not surprising, as the time resolution is much better in the ECG-based gating (1 ms) than in the presented list mode-based gating (50 ms). Besides, it is also not clear how cardiac arrhythmias would limit the performance of the proposed methods. These influences should be further investigated; perhaps a windowed Fourier analysis or a wavelet analysis may prove more valuable in these situations. Yet a clear correlation between ECG- and list mode-based ejection fractions was found in patients with high ${ }^{18}$ F-FDG uptake, and future refinement of the proposed methods may improve this situation. This might also be of interest in the context of combined PET/MRI scanners, in which ECG electrodes may interfere with magnetic fields. Nevertheless, it should be mentioned that list mode-based cardiac gating methods as presented here are inferior to ECG-based gating as the widely available gold standard.

Measured quantities in this study (motion shifts and wall thicknesses) by no means represent absolutely "true" values. Measured wall thicknesses should especially be treated carefully, both because of remaining partial-volume effects and because no attenuation correction was applied. The measured quantities just demonstrate one method's superior or inferior ability to capture respiratory motion as compared with another's ability.

Possible modifications of the presented list mode-based gating methods involve a more sophisticated rebinning method such as Fourier rebinning replacing the simple single-slice rebinning in the center-of-mass method, leading to a better axial classification of coincidences, although at the cost of processing time (19). Additionally, coincidences that are known to have originated outside the myocardium may be excluded from the analysis, thereby improving the noise properties of the raw gating signal.

A clear advantage of event-based methods is the fact that no additional equipment (e.g., sensors, video cameras, infrared cameras, and signal processing computers) is necessary to obtain gated scans, thus reducing costs and potential sources of errors. Additionally, the event-based methods are fast, as typical processing times to generate the respiratory curve from the list mode file were on the order of a few minutes on a quad-core workstation, depending on the actual activity inside the field of view and scanning duration. Bundschuh et al. recently proposed a gating method that is based on determining the respiratory motion of objects such as tumors by reconstructing short time frames, defining small volumes of interest containing these structures, and computing the craniocaudal motion of the center of the volume of interest (20). This method is similar to the center-of-mass method presented here; however, the processing of list mode data is extremely time-consuming as every frame has to be reconstructed before the center of mass is computed, whereas the centerof-mass method computes the center of mass on sinogram slices without prior reconstruction. One can even think of implementing the sensitivity and center-of-mass methods for real-time applications, obtaining a respiratory curve during the list mode scan; however, the filtering step necessary to get smooth curves has to be done afterward.

Further studies are needed to investigate if and how the proposed methods can be adopted for imaging of tumors close to the diaphragm, as these are known to be affected by respiratory motion. Besides better quantification of PET uptake values because of improved attenuation correction and less blurring, tumors can be located more precisely during the respiratory cycle when gating techniques are 
applied $(9,20)$. This improvement could be an advantage in radiotherapy, leading to highly precise radiation of tumor tissue. First results (not shown here) using list mode-based gating demonstrated their potential value also in the field of tumor imaging.

Future work will have to determine whether event-based methods can also be applied to tracer kinetic studies, for example, PET perfusion studies using $\mathrm{H}_{2}{ }^{15} \mathrm{O}$ or ${ }^{13} \mathrm{NH}_{3}$. It is clear that these methods have to be modified for this scenario, as in this case changes in total counts (measured in the sensitivity method) or shifts in axial center of mass (measured in the center-of-mass method) are correlated not only to respiratory motion or heart contractions but also to tracer kinetics.

One general drawback of PET gating is the loss of statistics in every PET gate, as can be seen in Supplemental Figure 2A. Although this loss may not be a problem in cases that have high tracer uptake in the heart, gated low-uptake scans really suffer from limited statistics, especially if the scans are compared with images obtained using slow CT scans for PET attenuation correction instead of PET gating to reduce attenuation correction artifacts. However, gating of PET data may just be the first step in a motion correction algorithm (21). The idea is to use the gated PET images to model a motion field between the different respiratory gates. This motion field can then be applied to correct for the respiratory motion and obtain motion-free PET images without loss of statistics. Several algorithms have already been investigated (rigid-body transformations (22), affine transformation models (23), optical flow-based methods $(24,25)$ ), and event-driven gating methods might prove to be a valuable and easily implemented base for future development.

\section{CONCLUSION}

Emission event-driven methods for respiratory gating were successfully used in a list mode-based cardiac PET/ CT feasibility study. Their performance was comparable to that of conventional video-based tracking of respiratory motion. Additionally, cardiac gating information can be obtained in scans with high tracer uptake. The event-driven center-of-mass-based method resulted in the best performance for respiratory and cardiac gating. List mode-based gating methods do not require additional hardware and can easily be applied in cardiac PET/CT.

\section{ACKNOWLEDGMENTS}

We thank Anika Brunegraf for technical assistance and Felix Range for patient acquisition. This study was supported by the Deutsche Forschungsgemeinschaft, Sonderforschungsbereich 656-Molecular Cardiovascular Imaging (SFB 656, projects $\mathrm{C} 2$ and $\mathrm{B} 3$ ), and a research grant to the European Institute of Molecular Imaging from Siemens Medical Solutions.

\section{REFERENCES}

1. Beyer T, Townsend DW, Brun T, et al. A combined PET/CT scanner for clinical oncology. J Nucl Med. 2000;41:1369-1379.

2. Kinahan PE, Townsend DW, Beyer T, Sashin D. Attenuation correction for a combined 3D PET/CT scanner. Med Phys. 1998;25:2046-2053.

3. Lang N, Dawood M, Büther F, Schober O, Schäfers M, Schäfers K. Organ movement reduction in PET/CT using dual-gated list mode acquisition. Z Med Phys. 2006;16:93-100.

4. Klein GJ, Reutter BW, Ho MW, Reed JH, Huesman RH. Real-time system for respiratory-cardiac gating in positron tomography. IEEE Trans Nucl Sci. 1998;45:2139-2143.

5. Nehmeh SA, Erdi YE, Ling CC, et al. Effects of respiratory gating on reducing lung motion artifacts in PET imaging of lung cancer. Med Phys. 2002;29:366371.

6. Dawood M, Büther F, Lang N, Schober O, Schäfers KP. Respiratory gating in positron emission tomography: a comparison of different gating schemes. Med Phys. 2007;34:3067-3076.

7. McKibben CK, Reo NV. A piezoelectric respiratory monitor for in vivo NMR. Magn Reson Med. 1992;27:338-342.

8. Boucher L, Rodrigue S, Lecomte R, Benard F. Respiratory gating for 3-dimensional PET of the thorax: feasibility and initial results. J Nucl Med. 2004;45:214-219.

9. Rietzel E, Chen GTY, Choi NC, Willet CG. Four-dimensional image-based treatment planning: target volume segmentation and dose calculation in the presence of respiratory motion. Int J Radiat Oncol Biol Phys. 2005;61:15351550

10. DeFronzo RA, Tobin JD, Andres R. Glucose clamp technique: a method for quantifying insulin secretion and resistance. Am J Physiol. 1979;237:E214E223.

11. He J, O'Keefe GJ, Jones G, et al. Evaluation of geometrical sensitivity for respiratory motion gating by GATE and NCAT simulation. Conf Proc IEEE Eng Med Biol Soc. 2007;1:4165-4168.

12. Daube-Witherspoon ME, Muehllehner G. Treatment of axial data in threedimensional PET. J Nucl Med. 1987;28:1717-1724.

13. Wink N, Panknin C, Solberg TD. Phase versus amplitude-based sorting of 4D-CT data. J Appl Clin Med Phys. 2006;7:77-85.

14. Stegger L, Lipke CS, Kies P, et al. Quantification of left ventricular volumes and ejection fraction from gated ${ }^{99 \mathrm{~m} T c-M I B I}$ SPECT: validation of an elastic surface model approach in comparison to cardiac magnetic resonance imaging, 4D-MSPECT and QGS. Eur J Nucl Med Mol Imaging. 2007;34:900-909.

15. Stegger L, Biedenstein S, Schäfers KP, Schober O, Schäfers M. Elastic surface contour detection for the measurement of ejection fraction in myocardial perfusion SPECT. Eur J Nucl Med. 2001;28:48-55.

16. Lagerwaard FJ, Van Sornsen de Koste JR, Nijssen-Visser MR, et al. Multiple "slow" CT scans for incorporating lung tumor mobility in radiotherapy planning. Int J Radiat Oncol Biol Phys. 2001;51:932-937.

17. Pan T, Mawlawi O, Luo D, et al. Attenuation correction of PET cardiac data with low-dose average CT in PET/CT. Med Phys. 2006;33:3931-3938.

18. Koch N, Liu HH, Starkschall G, et al. Evaluation of internal lung motion for respiratory-gated radiotherapy using MRI: part I-correlating internal lung motion with skin fiducial motion. Int J Radiat Oncol Biol Phys. 2004;60:14591472 .

19. Defrise M, Kinahan PE, Townsend DW, Michel C, Sibomana M, Newport D. Exact and approximate rebinning algorithms for 3D PET data. IEEE Trans Med Imaging. 1997;16:145-158.

20. Bundschuh RA, Martínez-Moeller A, Essler M, et al. Postacquisition detection of tumor motion in the lung and upper abdomen using list mode PET data: a feasibility study. J Nucl Med. 2007;48:758-763.

21. Schäfers KP, Dawood M, Lang N, Büther F, Schäfers M, Schober O. Motion correction in PET/CT. Nuklearmedizin. 2005;5a(suppl):S46-S50.

22. Livieratos L, Stegger L, Bloomfield PM, Schäfers K, Bailey DL, Camici PG. Rigid-body transformation of list mode projection data for respiratory motion correction in cardiac PET. Phys Med Biol. 2005;50:3313-3322.

23. Lamare F, Cresson T, Savean J, Cheze Le Rest C, Reader AJ, Visvikis D. Respiratory motion correction for PET oncology applications using affine transformation of list mode data. Phys Med Biol. 2007;52:121-140.

24. Dawood M, Lang N, Jiang X, Schäfers KP. Lung motion detection on respiratory gated 3D PET/CT images. IEEE Trans Med Imaging. 2006;25:476-485.

25. Dawood M, Büther F, Jiang X, Schäfers KP. Respiratory motion correction in 3-D PET data with advanced optical flow algorithms. IEEE Trans Med Imaging. 2008;27:1164-1175. 